\title{
Clinico-pathological correlations in neurodegeneration
}

\author{
Kurt A. Jellinger · Gabor G. Kovacs
}

Published online: 29 June 2011

(C) Springer-Verlag 2011

During the last years, Acta Neuropathologica has published a number of cluster fascicles focused on special neuropathological subjects, e.g., Alzheimer, Lewy body and prion diseases, brain tumor diagnosis, and animal models of nervous system diseases. Knowledge about clinicopathological correlations in neurodegenerative disorders has been widened, but also obscured by the rapid progress of clinical and basic sciences in detecting essential molecular and genetic backgrounds of these devastating disorders, leading to considerable changes in their traditional classifications and demonstrating multiple, often clinically similar, but biologically different subgroups. The classification of neurodegenerative diseases, previously based on the anatomical systems involved, has progressively been replaced by biologically and genetically defined groups, both promoting and obscuring the clinicians' views and making exact clinical diagnoses occasionally difficult without molecular pathological background. Neuropathologists as clinical neuroscientists are at the forefront of correlating histological and molecular changes in the brain with neurological features. Accordingly, the idea of reviewing the clinico-pathological correlations of the major neurodegenerative diseases, which should be of interest to both clinicians and basic neuroscientists, was born at the XVIIth International Congress of Neuropathology in Salzburg, Austria, in September 2010 .

K. A. Jellinger $(\bowtie)$

Institute of Clinical Neurobiology,

Kenyongasse 18, 1070 Vienna, Austria

e-mail: kurt.jellinger@univie.ac.at

G. G. Kovacs

Institute of Neurology,

Medical University of Vienna, Vienna, Austria
Manuel F. Casanova and colleagues present a critical review of the neuroimaging and morphological correlates of behavioral and psychological symptoms in dementia (BPSD), a subject hitherto rarely discussed in this global form. The review of the literature indicates that BPSD are the expression of regional rather than diffuse brain pathology: psychotic symptoms in demented patients are preferentially associated with the involvement of frontal and/or limbic regions, visual hallucinations with damage to the occipital lobes, apathy with the anterior cingulate gyrus, while white matter lesions appear to be significant for depression. However, BPSD may not be explained by neuropathological findings alone, but are influenced by genetic, neurochemical, environmental, personality, social, and other factors that need further elucidation.

Keith A. Josephs and coworkers, based on the pooled data from a number of large clinico-pathological studies, review the neuropathological background of phenotypical variability in frontotemporal dementias (FTD) that currently are classified into three main groups based on the major protein deposits in the brain; frontotemporal lobar degeneration (FTLD)-tau, -TDP, and -FUS. These groups and their specific pathology underly a number of clinical syndromes and the relationships of which are critically discussed. Although strong relationships were identified between FTD with motor neuron disease and FTLD-TDP; semantic dementia and FTLD-TDP, FTLD-tau, and progressive supranuclear palsy (PSP); and the corticobasal syndrome with FTLD-tau, other clinico-pathological relationships are still not clear, although there are some associations between clinical phenotypes and FTLD pathologies. While there remain diagnostic challenges, clinical diagnosis may often predict molecular pathology.

Richard Reynolds' group, based on the broad experience of the UKMS Tissue Bank, present a profound and critical 
review of the neuropathological basis of clinical progression in multiple sclerosis (MS), first describing the pathogenetic mechanisms during early stages and the neuroimaging and pathological substrates of clinical progression. They further review the clinico-pathological correlates in progressive MS including axonal, white and gray matter pathologies, remyelination and disability, the relations between cortical pathology, meningeal inflammation, neuronal loss, and clinical progression, and finally discuss benign MS. Convincing data support the suggestion that neurodegeneration leading to extensive gray matter pathology is important in the progression of clinical deficits. Considering the paucity of studies correlating the pathological features with clinical milestones in progressive MS, this paper is an important review of the contribution of the various types of pathology to the increasing neurological deficit in MS.

Reinhold Schmidt and coworkers extensively review the heterogeneity of age-related white matter changes in the brain, their neuroimaging correlates, risk factors and progression, and in particular the neuropathological correlates and molecular bases of these lesions. One has to distinguish punctate, early confluent and confluent types, the first showing a low tendency to progression, while the others progress rapidly. Although the basic mechanisms of sporadic age-related white matter changes are not fully understood, recent microarray and genome-wide approaches have provided insights into their causative pathways, including a locus on chromosome 17q25, cell-cycle dysregulation, proteolysis, ion transport mechanisms, and possibly ApoE, among others. Finally, age-related white matter changes using modern neuroimaging techniques are discussed, but further pre- and postmortem correlative studies using new imaging techniques are warranted.

Glenda M. Halliday and colleagues review the neuropathology underlying phenotypical variability in patients with synucleinopathies, featured by aggregates of abnormal $\alpha$-synuclein ( $\alpha \mathrm{Syn})$. They include Parkinson disease (PD), dementia with Lewy bodies (DLB) characterized by neuronal and neuritic deposits (Lewy bodies and neurites), multiple system atrophy with oligodendroglial $\alpha$ Syn deposits, and a large number of neurodegenerative disorders, including incidental Lewy body disease, a preclinical form of PD. However, Lewy pathology also occurs in Alzheimer disease (AD), in particular with the involvement of the amygdala, in neuroaxonal dystrophies, and is related to mutations of the $\beta$-glucosidase gene. Although there still is a low clinical sensitivity to identify DLB and related syndromes, recent clinico-pathologic studies indicate variable clinical features to occur in the different pathological forms of this syndrome. The recognition of the heterogeneity within the synucleinopathy syndromes is important for the identification of factors involved in changing their clinical and pathological phenotype.

As guest editors for this cluster, we are grateful to all the authors who have given their time and efforts to produce, what is hoped to be an informative and well-accepted series of review articles about difficult clinico-pathological problems in neurodegenerative disorders. They may not only provide information about some of the hitherto unclear or underestimated issues, but also should promote future research efforts to clarify several unanswered questions about the clinico-pathological correlations of these deleterious disorders. This needs new strategies for early and accurate diagnosis, better genetic and molecular workup, as well as deeper insights into their pathogenic cascade that influences the clinical course and outcome. All these efforts depend on close co-operation between clinicians, neuroscientists, geneticists, public health institutions, caregivers, and sponsors. What is presently puzzling and unclear will hopefully be unveiled by future neurobiological research to overcome the increasing burden of neurodegenerative and dementing disorders, and to promote effective preventive and treatment strategies. This cluster may help in resolving some of the secrets related to these disorders in the near future. 